\title{
IMPACTO DEL SEGUIMIENTO FARMACOTERAPÉUTICO EN LA CALIDAD DE VIDA RELACIONADA A LA SALUD DE PACIENTES CON HIPERTENSION ARTERIAL
}

\author{
Impact of pharmacotherapy follow up in the health-related quality of life of patients with \\ hypertension
}

\author{
Liana K. Ayala L ${ }^{1}$, Kelya Condezo M¹, José R. Juárez E² \\ ${ }^{1}$ Centro Médico Naval "Cirujano Mayor Santiago Távara” \\ ${ }^{2}$ Facultad de Farmacia y Bioquímica - Universidad Nacional Mayor de San Marcos
}

\section{RESUMEN}

Se evaluó el impacto de la intervención del farmacéutico en la calidad de vida relacionada a la salud (CVRS) de pacientes con hipertensión arterial esencial (HTA), que acuden a los consultorios externos del servicio de cardiología y reciben sus medicamentos a través de la Farmacia de Pacientes Crónicos del Centro Médico Naval "Cirujano Mayor Santiago Távara", durante un periodo de seis meses, utilizando el método Dáder de seguimiento farmacoterapéutico (SFT). Se incluyeron en el estudio 14 pacientes de 40 años a más, $71,4 \%$ varones y $28,6 \%$ mujeres, sin otra patología crónica asociada. Se empleó el cuestionario específico MINICHAL para determinar la CVRS al inicio del estudio, durante y después de la intervención. Se detectaron 29 problemas relacionados con los medicamentos (PRM): 66\% relacionados con la necesidad, $24 \%$ con la efectividad y $10 \%$ con la seguridad. Se resolvieron 24 PRM (75\%), lo que demuestra el grado de aceptación de las recomendaciones farmacéuticas para eliminar las causas prevenibles de los PRM y justifican la incorporación del SFT como parte esencial de la función del farmacéutico hospitalario. La comunicación de las intervenciones se hicieron en mayor porcentaje entre farmacéutico-paciente (87,5\%), tanto verbal como escrita, y farmacéutico-paciente-médico (12,5\%) en forma escrita. Los resultados mostraron al $57 \%$ de los pacientes con presión arterial controlada al culminar el estudio, en comparación con el $42 \%$ al inicio de éste. Se concluye que, la CVRS se ve influenciada positivamente por la intervención del farmacéutico en el SFT.

Palabras clave: Calidad de vida, calidad de vida relacionada a la salud, seguimiento farmacoterapéutico, hipertensión arterial.

\section{SUMMARY}

Was evaluated the impact that generates the intervention of the pharmacist in the health-related quality of life HRQL patients with diagnosis of essential arterial hypertension (AHT). They was from external doctor's offices of the Cardiology Service and they received their medicines through the Chronic Patients Drugstore of the Navy Medical Center "Cirujano Mayor Santiago Távara”, during a period of six months, using the Dáder method of pharmacotherapy follow-up (PTF). 14 patients of 40 years old to more were included in the study, $71,4 \%$ were men and $28,6 \%$ women without another associate chronic pathology. Specific MINICHAL questionnaire was used in order to determine the HRQL to the beginning of the study, during and later of pharmaceutical intervention. It was detected 29 drug-related problems (DRP): 66\% of them related mainly to the necessity, $24 \%$ to effectiveness and $10 \%$ to security. Were solved 24 drug related problems (DRPs), $(75 \%)$ that demonstrates the degree of acceptance of the pharmaceutical recommendations to eliminate the causes of DRPs and justify the incorporation of the PTF as essential part in the function of the hospital pharmacist. The communication of the interventions were done in major percentage between pharmacist-patient $(87,5 \%)$, using both the verbal form as the written one, and pharmacist-patient-phycicians (12,5\%) using the written form. At the end of the study, $57 \%$ of the patients had controlled their arterial pressure, in comparison with $42 \%$ of the patients at the beginning of the study. Was conclude that the HRQL is positively influenced by pharmacist intervention through PTF.

Keywords: Life quality health-related quality of life, pharmacotherapy follow-up, arterial hypertension.

\section{INTRODUCCIÓN}

$\mathrm{E}$ n las Américas, 144 millones de personas viven con hipertensión arterial (HTA) ${ }^{(1)}$. En la región de América Latina y el Caribe se estima que entre el 8 y $35 \%$ de su población adolece de HTA, lo que de alguna forma se confirma con el estudio realizado por la Sociedad Peruana de Cardiología en la ciudad de Lima en febrero del 2004, donde se muestra una prevalencia de HTA de $23 \%$, con ligera diferencia entre hombresy mujeres, de $26 \%$ y $20 \%$ respectivamente, tendencia que se observa también en otros estudios ${ }^{(2)}$.

El seguimiento del tratamiento farmacológico 
Tabla 1. Distribución de pacientes por género y grupos etáreos.

\begin{tabular}{|c|c|c|c|c|c|}
\hline \multirow{2}{*}{ Género } & \multicolumn{4}{|c|}{ Grupos etáreos } & \multirow{2}{*}{ Total\% } \\
\hline & $40-49$ & $50-59$ & $60-69$ & $>70$ & \\
\hline Varones & --- & 4 & 4 & 2 & 71,43 \\
\hline Mujeres & 1 & 1 & 2 & --- & 28,57 \\
\hline $\begin{array}{l}\text { Porcentaje } \\
\text { por grupo }\end{array}$ & 7 & 36 & 43 & 14 & \\
\hline
\end{tabular}

Tabla 2. Fármacos más utilizados.

\begin{tabular}{cc}
\hline Fármaco & \% de uso \\
\hline Amlodipino & 21 \\
ASA & 21 \\
Atenolol & 18 \\
Hidroclorotiazida/Amilorida & 18 \\
Irbezartan & 7 \\
Lisinopril & 4 \\
Quinapril & 7 \\
Verapamilo & 4 \\
\hline
\end{tabular}

en pacientes con HTA tiene como objetivo el cumplimiento de la farmacoterapia a fin de lograr el mantenimiento de la presión arterial en niveles óptimos para el paciente. Para conseguirlo, la actuación del farmacéutico, bajo el concepto de la Atención Farmacéutica, va encaminada a la detección y prevención de problemas relacionados con los medicamentos (PRM) y su posterior resolución mediante las intervenciones oportunas ${ }^{(3)}$.

El concepto de calidad de vida relacionada a la salud (CVRS), agrupa elementos propios del individuo y otros externos al mismo, pero que interaccionan con él y pueden llegar a cambiar su estado de salud. Es un concepto que refleja las conductas físicas, emocionales y sociales, y está relacionado con el estado de salud previo y actual. Abarca áreas de función física, somática, estado psicológico y relación social. La medida CVRS es central para describir el impacto de enfermedades, tratamientos u otras acciones ${ }^{(4,5)}$.

Por tal motivo, en el presente trabajo nos trazamos como objetivo evaluar el impacto que genera la intervención del farmacéutico en la calidad de vida relacionada a la salud en pacientes con HTA esencial.

\section{MATERIAL Y MÉTODOS}

Se trata de un estudio de tipo experimental, longitudinal y prospectivo, en el que se incluyeron catorce pacientes con diagnóstico de HTA esencial que acuden a los consultorios externos del servicio de cardiología y reciben
Tabla 3. PRM detectados y resueltos.

\begin{tabular}{|c|c|c|}
\hline \multirow{2}{*}{ PRM } & \multicolumn{2}{|c|}{ Porcentaje } \\
\hline & Detectados & Resuelto \\
\hline Necesidad & 66 & 75 \\
\hline Efectividad & 24 & 16,67 \\
\hline Seguridad & 10 & 8,33 \\
\hline
\end{tabular}

sus medicamentos a través de la Farmacia de Pacientes Crónicos del Centro Médico Naval "Cirujano Mayor Santiago Távara" y que cumplían los criterios de selección; todos ellos firmaron la hoja de consentimiento informado.

Luego a cada paciente se le pidió que desarrolle el cuestionario de CVRS MINICHAL, validado. Los 14 pacientes fueron divididos aleatoriamente en grupo de estudio (7) y grupo control (7); todos participaron los seis meses establecidos de seguimiento. A cada uno de los pacientes se le elaboró su historia farmacoterapéutica, además de disponer de otras herramientas, tales como: Tabla de registro diario de la presión arterial, Tabla de registro mensual de peso, Tabla de registro diario para el conocimiento de los hábitos higiénico dietéticos; se dispuso de material informativo para el paciente: trípticos, afiches, etc. Para la comunicación con el médico tratante se emplearon cartas informativas.

Siguiendo la metodología DADER, se cumplió cada una de las etapas que ésta considera, y se procedió a la recolección de datos.

\section{RESULTADOS Y DISCUSIÓN}

Los porcentajes, por género, en el grupo de pacientes seleccionado para el estudio: $71,43 \%$ de varones y $28,57 \%$ de mujeres, concuerda con datos encontrados en otros estudios realizados en nuestro país ${ }^{(2,6)}$. El $7 \%$ de los pacientes estuvieron comprendidos entre 40 y 49 años, el $36 \%$ entre 50 y 59 , el $43 \%$ entre 60 y 69 y el $14 \%$ de 70 años a más; evidenciándose un incremento de pacientes con hipertensión arterial en el grupo etáreo de 60 a 69 
Tabla 4. Valores obtenidos en la medición de la CVRS

\begin{tabular}{|c|c|c|c|c|}
\hline \multirow{2}{*}{ Paciente } & \multicolumn{2}{|c|}{ Mediciones grupo control } & \multicolumn{2}{|c|}{ Mediciones grupo de intervención } \\
\hline & 10 (Mes 0) & $2^{\circ}($ Mes 6) & 1o (Mes 0) & $2^{\circ}($ Mes 6) \\
\hline $\mathrm{P}_{1}$ & 7 & 10 & 9 & 4 \\
\hline $\mathrm{P}_{2}$ & 11 & 12 & 4 & 1 \\
\hline $\mathrm{P}_{3}$ & 12 & 18 & 0 & 0 \\
\hline $\mathrm{P}_{4}$ & 4 & 5 & 8 & 5 \\
\hline$P_{5}$ & 22 & 20 & 15 & 11 \\
\hline $\mathrm{P}_{6}$ & 4 & 6 & 20 & 10 \\
\hline $\mathrm{P}_{7}$ & 1 & 6 & 7 & 3 \\
\hline Promedio & 8,571 & 9,857 & 9,000 & 4,714 \\
\hline
\end{tabular}

años de edad (Tabla 1), coincidente con estudios que revelan un incremento en la prevalencia de HTA a medida que avanza la edad ${ }^{(7)}$.

La relación de PRM/paciente, en el grupo de seguimiento, encontrada en este estudio fue de 4,14; mientras que en otros estudios se registran porcentajes relativamente inferiores, como en el estudio de Dualde que reporta $0,82^{(3,8,9)}$. Esta diferencia podría evidenciar una mayor necesidad de intervenciones farmacéuticas en este tipo de pacientes. Cabe mencionar que el PRMi representó el mayor porcentaje de los encontrados $(66 \%)$, esto fundamentalmente por la no disponibilidad del medicamento en farmacia al momento de requerimiento del paciente.

Las intervenciones del farmacéutico, medidas a través de la comunicación, se hicieron en mayor porcentaje entre farmacéutico-paciente $(87,5 \%)$ empleando tanto la forma verbal como la escrita, y en segundo lugar la comunicación farmacéuticopaciente-médico $(12,5 \%)$, la forma empleada para este caso fue la escrita.

Para la medición de la CVRS, se utilizó un instrumento previamente validado con la finalidad de asegurar su medición en la población sujeta al estudio. Es así que se realizó un análisis secundario que tuvo como objetivo analizar la influencia de las variables sociodemográficas y clínicas sobre la CVRS de los pacientes hipertensos y evaluar el efecto del tratamiento antihipertensivo sobre dicha CVRS ${ }^{(10)}$. Para la validación se empleó un formulario de entrevista al paciente, que fue aplicado luego de que éste desarrollara el cuestionario MINICHAL. Este procedimiento se realizó en los primeros 10 pacientes que acudieron a la Farmacia de Pacientes Crónicos y que cumplían los criterios de selección. El formulario constó de siete preguntas, de las cuales cuatro correspondían a preguntas cerradas y las restantes a preguntas abiertas, estas últimas estaban formuladas en función a las respuestas de las preguntas cerradas.

En la tabla 4 se muestran los valores promedio obtenidos en las diferentes entrevistas tanto en el grupo control como en el de seguimiento con una media inicial de 8,57 para el grupo control y 9 para el grupo de seguimiento. Estos resultados concuerdan con otros estudios que señalan que los pacientes con HTA tienen una pobre calidad de vida en comparación a la población en general o con pacientes que no tienen condiciones crónicas ${ }^{(11,12)}$. Esta percepción está relacionada principalmente con la presencia de síntomas que pueden derivar de la enfermedad (incremento anormal de la presión arterial) o de efectos nocivos del tratamiento. Sin embargo, a los seis meses y, a medida que el seguimiento farmacoterapéutico fue aplicado (Grupo de intervención), se evidenció un cambio positivo en la calidad de vida de los pacientes con HTA, pues mientras que en el grupo control se obtiene una media de 9,85, que llega hasta 11 al sexto mes indicando una disminución en su CVRS, en el grupo de intervención fue de 4,71, lo que señala un aumento en la CVRS. Estos cambios están relacionados con la prevención o disminución de los síntomas -solucionando PRM reales o potenciales-, la mejora en el cumplimiento de la terapia farmacológica y no farmacológica, así como con la motivación de mejora en la salud del paciente.

En lo referente a los controles de presión arterial, los resultados muestran un $57 \%$ de los pacientes con valores $<140$ y $<90 \mathrm{mmHg}$ al final del estudio, en comparación con el $43 \%$ al inicio del estudio; además se logró que un paciente disminuyera los valores de presión arterial sistólica (PAS) desde un rango de $>160$ a $140 \mathrm{mmHg}$, mientras que otro paciente alcanzó los valores óptimos de 
presión arterial (PA), es decir $<\mathbf{1 2 0} /<80 \mathrm{mmHg}$ (Tabla 2).

\section{CONCLUSIONES}

La intervención del farmacéutico mediante el seguimiento farmacoterapéutico, además de permitir colaborar en el control y mantenimiento de los niveles de presión arterial, contribuye a mejorar la calidad de vida relacionada con la salud en los pacientes con HTA esencial.

\section{REFERENCIAS BIBLIOGRÁFICAS}

1. Foro Farmacéutico de las Américas (FFA). Organización Panamericana de la Salud (OPS). Washington DC, Agosto 2004.

2. Oficina General de Epidemiología. Boletín Epidemiológico SEl $\mathrm{N}^{\circ}$ 35, Ministerio de Salud. Lima 2004; XIII(40).

3. Dualde VE. Control y seguimiento de medicación en pacientes hipertensos. Pharmaceutical Care España 1999; 1: 28-34.

4. Paredero VM, Arrébola-López M, Berga-Fauria C, Pañella-Agusti $F$ y Gómez-Moya B. Impacto en la calidad de vida. Angiología 2003; 55: 250-9.

5. Bruce B, Fries JF. The Stanford Health Assesment Questionnaire: Dimensions and Practical Applications. Health and quality of life outcomes 2003, 1:20.

6. Organización Panamericana de la Salud. Análisis de la situación de salud en el Perú. Lima, 2002.
7. Regulo C. Epidemiología de la hipertensión arterial en el Perú. Acta Med Per 2006; 23(2): 69-75.

8. Flores L, Segura C, Quesada MS y Hall V. Seguimiento Farmacoterapéutico con el método Dáder en un grupo de pacientes con hipertensión arterial. Seguimiento Farmacoterapéutico 2005; 3(3): 154-57.

9. Álvarez A, Juárez EJ. Atención farmacéutica en pacientes con insuficiencia cardiaca en el Hospital Nacional Guillermo Almenara Irigoyen - EsSALUD (Perú). Revista de la O.F.I.L. 2002; 12(2): 32-38.

10. Roca-Cusachs A et al. Relación entre variables clínicas y terapéuticas y calidad de vida relacionada con la salud en pacientes con hipertensión arterial. Estudio MINICHAL. Med Clin (Barc) 2003; 121(1):12-7.

11. Bardage C, Isacson DGL. Hypertension and healthrelated quality of life: an epidemiological study in Sweden. J Clin Epidemiol 2001; 54:172-81.

12. Stewart AL, Greenfield S, Hays RD et al. Functional status and well-being of patients with chronic conditions: results from the medical outcomes study. JAMA 1989; 262:907-13.

Manuscrito recibido el: 15/11/2010

Aceptado para su publicación el: 18/o2/2011

\section{Correspondencia:}

Nombre: José Roger Juárez Eyzaguirre

Dirección: Jr. Puno 1002 - Lima 1 - Peru.

e-mail: jjuareze@unmsm.edu.pe 